\title{
Genetic risk for Alzheimer's disease is concentrated in specific macrophage and microglial transcriptional networks
}

\author{
Katherine E. Tansey ${ }^{1}$, Darren Cameron ${ }^{2}$ and Matthew J. Hill ${ }^{2,3^{*}}$ (1)
}

\begin{abstract}
Background: Genome-wide association studies of Alzheimer's disease (AD) have identified a number of significant risk loci, the majority of which lie in non-coding regions of the genome. The lack of causal alleles and considerable polygenicity remains a significant barrier to translation into mechanistic understanding. This includes identifying causal variants and the cell/tissue types in which they operate. A fuller understanding of the cell types and transcriptional networks involved in AD genetic risk mechanisms will provide important insights into pathogenesis.

Methods: We assessed the significance of the overlap between genome-wide significant AD risk variants and sites of open chromatin from data sets representing diverse tissue types. We then focussed on macrophages and microglia to investigate the role of open chromatin sites containing motifs for specific transcription factors. Partitioned heritability using LDscore regression was used to investigate the contribution of specific macrophage and microglia transcription factor motif-containing open chromatin sites to the heritability of AD.

Results: AD risk single nucleotide polymorphisms (SNPs) are preferentially located at sites of open chromatin in immune cells, particularly monocytes $\left(z\right.$ score $=4.43$; corrected $\left.P=5.88 \times 10^{-3}\right)$. Similar enrichments are observed for macrophages $\left(z\right.$ score $=4.10$; corrected $\left.P<2.40 \times 10^{-3}\right)$ and microglia $(z$ score $=4.34$, corrected $P=0.011)$. In both macrophages and microglia, AD risk variants are enriched at a subset of open chromatin sites that contain DNA binding motifs for specific transcription factors, e.g. SPI1 and MEF2. Genetic variation at many of these motif-containing sites also mediate a substantial proportion of AD heritability, with SPI1-containing sites capturing the majority of the common variant SNP-chip heritability (microglia enrichment $=16.28$, corrected enrichment $P=0.0044$ ).

Conclusions: AD risk alleles plausibly operate in immune cells, including microglia, and are concentrated in specific transcriptional networks. Combined with primary genetic association results, the SPI1 and MEF2 transcriptional networks appear central to AD risk mechanisms. Investigation of transcription factors targeting AD risk SNP associated regulatory elements could provide powerful insights into the molecular processes affected by AD polygenic risk. More broadly, our findings support a model of polygenic disease risk that arises from variants located in specific transcriptional networks.
\end{abstract}

Keywords: Alzheimer's disease, GWAS, Transcription factor, Non-coding variation

\footnotetext{
* Correspondence: hillm6@cardiff.ac.uk

${ }^{2}$ MRC Centre for Neuropsychiatric Genetics and Genomics, Division of Psychological Medicine and Clinical Neurosciences, School of Medicine, College of Biomedical and Life Sciences, Cardiff University, Hadyn Ellis Building, Maindy Road, Cardiff CF24 4HQ, UK

${ }^{3}$ UK Dementia Research Institute, Cardiff University, Hadyn Ellis Building, Maindy Road, Cardiff CF24 4HQ, UK

Full list of author information is available at the end of the article
} 


\section{Background}

Genome-wide association studies (GWAS) of Alzheimer's disease $(\mathrm{AD})$ have identified multiple loci containing common variant risk alleles [1]. These findings offer new routes to understanding disease biology that could be used to design novel therapies. However, like other complex diseases and traits, the majority of these risk alleles are located in non-coding regions of the genome [2], making immediate functional interpretation difficult. Furthermore, at each locus the risk signal is often associated with multiple variants in strong linkage disequilibrium (LD), any of which could credibly be the causal variant(s). Nevertheless, analytical approaches, such as pathway analysis [3] and integration with chromatin annotations [4, 5], have begun to identify the cell types and processes that are likely to be disrupted by AD risk alleles. Strikingly, these complementary approaches have identified immune cells and pathways as the likely effectors of $\mathrm{AD}$ genetic risk. Despite these advances, the full repertoire of potentially causal cell types and the molecular mechanisms through which AD risk variants operate have yet to be fully investigated. This includes the identification of functional variants at genome-wide significant risk loci as well as the mechanisms through which polygenic risk operates.

Of these approaches, integration of genetic association data with the growing amount of functional genomic annotations (e.g. ENCODE [6] and Roadmap Epigenomics [7]) have the potential to identify: (1) causal non-coding risk alleles, (2) the mechanisms by which they operate and (3) the cell types in which they function $[8,9]$. While risk alleles at genome-wide significant loci represent robust findings suitable for biological characterisation, it is now known that thousands of variants throughout the genome contribute to disease heritability [10]. Recently developed analytical methods, such as stratified LDscore regression [11, 12], can use these annotations to investigate the relevance of specific cell types to the heritability of a disease of interest, extending analysis beyond genome-wide significant loci to capture polygenic risk mechanisms.

Several technologies now exist for genome-wide identification of non-coding elements with regulatory potential. These range from the study of post-translational modifications of histones to the resolution of binding sites for specific transcription factors; collectively termed chromatin immunoprecipitation (ChIP). Methods that rely on discriminating local chromatin structure, such as DNaseseq [13] and assay for transposase-accessible chromatin using sequencing (ATAC-seq) [14], can identify potential transcription factor binding sites without the need for performing multiple transcription factor ChIP experiments. These open chromatin regions (OCRs) display a high degree of cell-type specificity, defining promoters of expressed genes as well as distal regulatory elements [13], and are enriched for DNA motifs recognised by transcription factors important for determining cell lineage and function [15]. Although the integration of chromatin annotations with GWAS results has been successful in identifying disease-relevant tissues [2, 8, 16], few, if any, have attempted to attribute genome-wide polygenic risk mechanisms to specific transcription factor networks.

We, therefore, reasoned that the integration of results from GWAS of AD with OCRs from multiple cell types would pinpoint disease relevant cell types and link AD genetic risk variants to specific transcriptional networks active in those cell types.

\section{Methods \\ Data processing}

DNase hypersensitivity sites (DHSs) and histone ChIP-seq peaks (H3K4me3, H3K4me1 and H3K27ac) were generated by the Roadmap Epigenomics Project [7]. Monocyte and macrophage DNase-seq data were generated by Blueprint (http://dcc.blueprint-epigenome.eu/\#/home). All data sets had been mapped to hg19 (GRCh37). Data were processed using BEDTools [17]. Cancer-derived cells lines present in the Epigenomics Roadmap data set were removed before further analyses. Microglia ATAC-seq data [18] were obtained from dbGaP Study Accession: phs001373.v1.p1. Data were aligned to hg19 (GRCh37) using bwa [19] and peaks were called using hotspot [20], following the protocol described by the Blueprint Consortium.

\section{Enrichment testing for the overlap between AD risk variants and open chromatin regions}

Genome-wide significant $\left(P<5 \times 10^{-}{ }^{8}\right)$ AD risk variants [(GWAS index single nucleotide polymorphisms (SNPs)] identified by Lambert et al. [1] were downloaded from the GWAS catalogue [21]. Variants located in the APOE and major histocompatibility complex (MHC) regions were excluded, resulting in 18 GWAS index SNPs. For the remaining GWAS index SNPs, 10,000 matched sets of variants were generated using SNPsnap [22], which matches SNPs based on allele frequency, number of SNPs in LD, distance to nearest gene and gene density. Variants in high LD $\left(r^{2}>0.8\right)$ with each SNP (GWAS index SNPs and matched sets) were extracted from the 1000 Genomes Project (phase 3). The resulting 10,001 SNP sets were then intersected with OCRs and histone peaks using BEDTools. The number of overlapping loci was calculated for each set and the deviation from the background matched sets was calculated as a $z$ score. $P$ values were calculated by direct observation of the number of background matched SNPs sets that exceeded the overlap of the GWAS index SNP set (minimum possible uncorrected $P$ value is therefore $\left.1 \times 10^{-4}\right)$. 


\section{De novo motif analysis and assignment to open} chromatin regions

Macrophage DHSs for the 16 data sets from the BLUEPRINT Project were merged to form a consolidated data set using BEDTools, run with default parameters. Microglia ATAC peaks for the 12 donors were similarly merged to form a consolidated set. The consolidated sets were then used as input for de novo motif discovery using HOMER, [23] with default parameters. The resulting motifs were then assigned to OCRs using the HOMER command findMotifs.pl with the '-find' option enabled.

\section{Partitioned heritability using LDscore regression}

LDscore regression $[11,12]$ was used to partition AD genetic heritability by motif-containing sites identified as being enriched at genome-wide significant loci (e.g. CEBPA, EGR1, MEF2A and SPI1 for macrophages), following the previously described methodology [12]. AD genome-wide associated results were downloaded from http://web. pasteur-lille.fr/en/recherche/u744/igap/igap_download.php, and only phase 1 data were used. The no-motif-containing set was included as a negative control. Sites were extended by \pm 500 base pairs, consistent with previous partitioning heritability studies [12]. LDscore files were made for each specific annotation of interest using the open source software available here: https://github.com/bulik/ldsc/wiki. The MHC region (chr6:26,000-34,000 kb) and APOE region (chr19:44,400-46,500 kb) were removed. The results remain significant with the inclusion of these regions (data not shown). Each annotation was added to the baseline model independently, creating five separate models. The baseline model includes 24 non-cell-specific annotations that cover a range of DNA features, such as coding, 3' untranslated region, promoter, intronic, H3K4me1 marks, H3K4me3 marks, H3K9ac marks, H3K27ac marks, DNase I hypersensitivity sites, chromHMM and Segway predictions, regions conserved in mammals, super-enhancers and FANTOM5 enhancers (please see Finucane et al. [12] for more information about the baseline model).

\section{Web resources}

\section{Software:}

LDscore: https://github.com/bulik/ldsc/wiki

HOMER: http://homer.ucsd.edu/homer/motif/index.html SNPsnap: https:/data.broadinstitute.org/mpg/snpsnap/ BEDTools: http://bedtools.readthedocs.io/en/latest/ Data availability:

Data generated by the Roadmap Epigenomics Project were downloaded from http://egg2.wustl.edu/roadmap/ data/byFileType/peaks/consolidated/broadPeak/DNase/

http://egg2.wustl.edu/roadmap/data/byFileType/peaks/ consolidated/broadPeak/

http://egg2.wustl.edu/roadmap/data/byFile'Type/peaks/ consolidated/narrowPeak/
Blueprint monocyte and macrophage DHSs were downloaded from http://ftp.ebi.ac.uk/pub/databases/ blueprint/data/homo_sapiens/GRCh37/

$\mathrm{AD}$ genome-wide associated results were downloaded from http://web.pasteur-lille.fr/en/recherche/u744/igap/ igap_download.php

1000 Genomes data were downloaded from http:// www.internationalgenome.org/about\#ProjectSamples

Microglia ATAC-seq data were obtained from: https:// www.ncbi.nlm.nih.gov/projects/gap/cgi-bin/study.cgi? study_id=phs001373.v1.p1

\section{Results}

Enrichment of $A D$ risk variants at DNase hypersensitivity sites across tissue/cell types

We assessed whether AD risk variants (index SNPs and variants in $\mathrm{LD}$ at $r^{2}>0.8$ ) were preferentially located at DHSs from a panel of 38 tissues profiled by the Roadmap Epigenomics Consortium [7]. Three cell/tissue types remained significant after correcting for all tests of enrichment (DNase and the three histone modifications) using the method described by Benjamini and Hochberg [24] (Fig. 1). Of these, two were immune cell types (primary haematopoietic stem cells G-CSF-mobilised, $z$ score $=4.75$, corrected $P=4.2 \times 10^{-3}$; and primary monocytes from peripheral blood, $z$ score 4.43 , corrected $\left.P=5.9 \times 10^{-3}\right)$. Several other immune cell types ranked highly in the analysis with four of the five most enriched tissue types being immune cells. However, these did not remain significant after correction for multiple testing. Only two brain samples, both foetal, were available in this DHS data set, and neither showed significant enrichment after correction for multiple testing $(z$ score $=2.63$ and 1.40, uncorrected $P=0.011$ and 0.140 ). Full details of the results for each sample can be found in Additional file 1: Table S1. To confirm our cell/tissue-type enrichments, we also performed enrichment analyses using regions marked by the histone modifications H3K27ac, H3K4me1 and H4K3me3. For all three histone modifications, the largest enrichment was observed in monocytes (Additional file 2: Table S2, Additional file 3: Table S3 and Additional file 4: Table S4). Although several immune cell types were significantly enriched across these analyses, only monocytes were significant in all four chromatin feature analyses.

\section{Enrichment of $A D$ risk variants at DNase hypersensitive sites in monocytes and macrophages}

Given that data generated from the Roadmap Epigenomics Consortium are derived from a limited number of donors, we sought to replicate these findings and test additional immune cell types. DNase hypersensitivity data from 16 macrophage and seven monocyte samples were available from the Blueprint Epigenome Project (http://dcc.blueprint-epigenome.eu/\#/home). Using these 


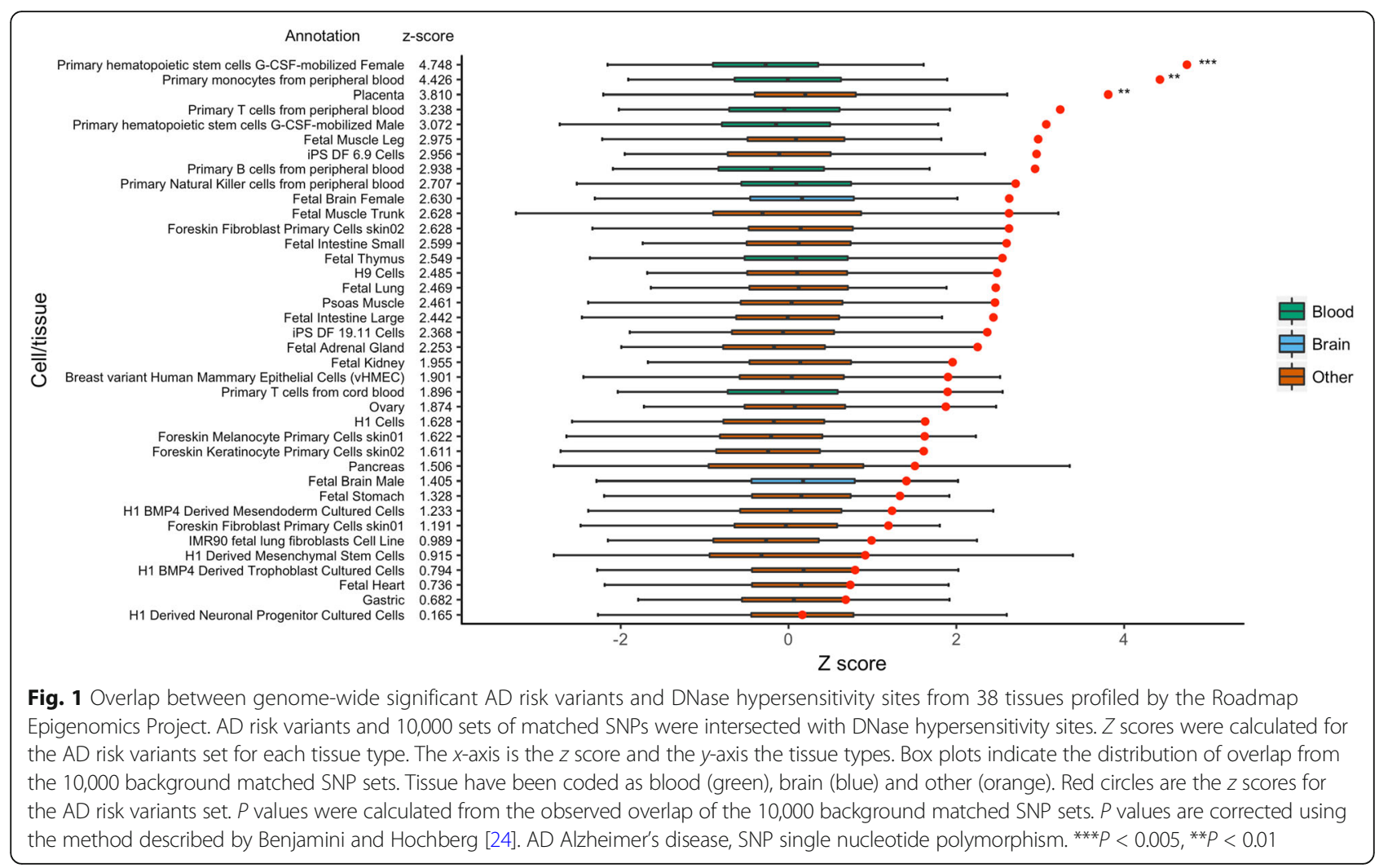

data, enrichment $z$ scores for the overlap with AD risk variants ranged from 3.00 to $5.07($ mean $=4.12)$ for the seven monocyte samples, and 1.98 to $5.32($ mean $=3.88)$ for the 16 macrophage samples (Fig. 2). In total, 14 of the 23 samples tested were significant after correction for multiple testing using the Bonferroni method to correct for 37 tests (35 monocyte/macrophage/microglia samples plus the two consolidated sets), replicating the enrichment of $\mathrm{AD}$ variants at immune cell DHSs, and identifying macrophages as a potential cell type affected by $\mathrm{AD}$ genetic risk.

To reduce inconsistencies arising from selecting individual donor samples, a consolidated set of macrophage DHSs was generated by merging the peaks from the 16 different data files. AD risk variants were similarly enriched at DHSs in this consolidated set $(z$ score $=$ 4.10, $P<1 \times 10^{-4}$, corrected $P<3.7 \times 10^{-3}$ ), with 13 of the 18 loci tested having at least one overlapping SNP (Fig. 3). At these 13 loci, the number of SNPs overlapping macrophage DHSs ranges from 1 to 11 (Additional file 5: Table S5 and Additional file 6: Figure S1), indicating multiple potential causal alleles. These loci contain genes with both overt immune cell functions (e.g. INPP5D) and no known immune-cell-specific activity (e.g. BIN1 and PICALM).

\section{Enrichment of $A D$ risk variants at open chromatin regions in microglia}

We obtained publicly available human microglia open chromatin (ATAC-seq) data from 12 donors [18] to investigate the role of the resident brain macrophage in $\mathrm{AD}$ genetic risk mechanisms. We observed enrichment $z$ scores ranging from 2.77 to 5.25 (mean $=4.07$ ). In total, nine donor samples were significant after Bonferroni correction for the 37 tests (35 monocyte/macrophage/ microglia samples plus the two consolidated sets). AD risk variants were also enriched at microglia ATAC-seq peaks using the consolidated peak set $(z$ score $=4.34$, corrected $P=0.011)$, with a total of 11 loci containing at least one SNP that overlapped an ATAC-seq peak (Fig. 3). Additional file 7: Table S6 contains a full list of overlapping SNPs and gene annotations.

\section{Enrichment of AD risk SNPs at open chromatin regions containing specific transcription factor motifs}

We further investigated the localisation of AD risk variants to specific subsets of macrophage and microglia OCRs defined by the presence of specific transcription factor DNA binding motifs. De novo motif analysis of the consolidated sets of macrophage DHS or microglia ATAC-seq peaks was performed using HOMER [23]. 

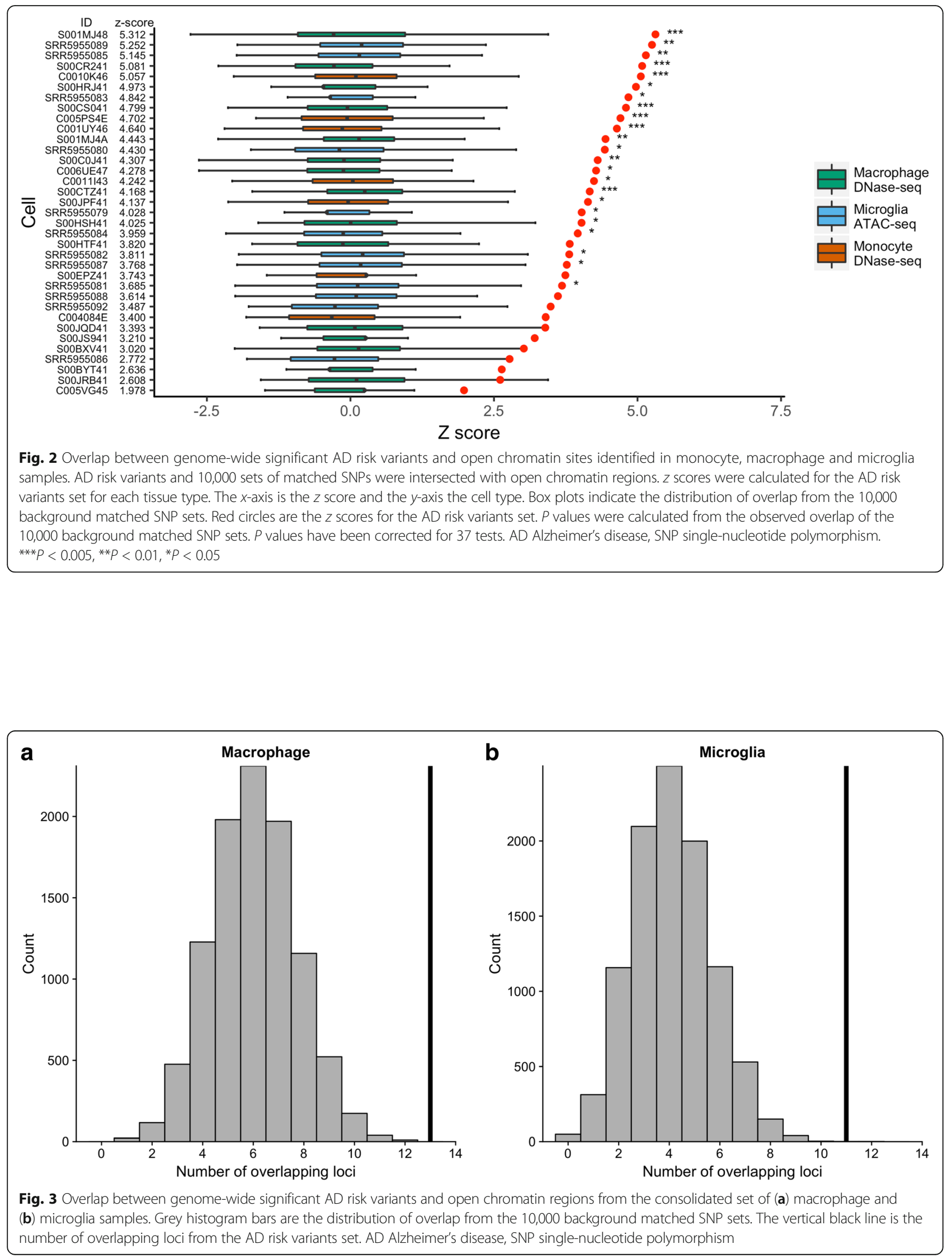
In the macrophage DHS, this identified 15 enriched motifs (Additional file 8: Table S7), including established regulators of immune cell function (e.g. SPI1 and NFKB). We then grouped DHSs according to the presence or absence of a motif for each of the 15 motifs identified, generating 16 subsets, one for each specific transcription factor motif and one with DHSs that lacked any of these motifs. Two motif sets were removed from the analysis as fewer than 1000 of the 10,000 background matched SNPs showed any overlap. AD risk variants were significantly enriched after correction for multiple testing using the Bonferroni method at DHSs containing the motifs SPI1 (PU.1) $(z$ score $=5.53$, corrected $\left.P<1.30 \times 10^{-3}\right)$, EGR1 $(z$ score $=4.40$, corrected $\left.P<1.30 \times 10^{-3}\right)$, MEF2A $(z$ score $=4.08$, corrected $P=$ 0.023 ) or CEBPA ( $z$ score $=3.68$, corrected $P=0.013$ ) (Fig. 4a). The SPI1 (PU.1) motif set captured all 13 of the loci that showed an overlap with the consolidated macrophage DHS set. The number of SNPs overlapping the SPI1 motif-containing DHS at each locus ranged from 1 to 7, implicating multiple potential causal SNPs.

De novo motif analysis of the microglia ATAC-seq peaks identified 19 motifs (Additional file 9: Table S8), including known lineage-determining factors such as SPI1 and IRF [25]. Sets of motif-containing peaks were then generated as described above. After intersecting with SNP data, one motif set was removed from the analysis as fewer than 1000 of the 10,000 background matched SNPs showed any overlap. AD risk variants were significantly enriched at ATAC-seq peaks containing motifs for RUNX $\left(z\right.$ score $=5.22$, corrected $\left.P<1.9 \times 10^{-3}\right)$, SPI1 (PU.1) $\left(z\right.$ score $=5.02$, corrected $\left.P<1.9 \times 10^{-3}\right)$ and Spdef $(z$ score $=3.80$, corrected $P=0.027)$ after correction for multiple testing using the Bonferroni method (Fig. 4b). Like the macrophages, the SPI1 (PU.1) set captured the largest number of loci, accounting for nine of the 11 overlaps identified using all ATAC-seq peaks in the consolidated set. We validated our motif-based findings using SPI1 (PU.1) ChIP-seq data from human microglia [18]. $\mathrm{AD}$ risk variants were significantly enriched at these experimentally identified SPI1 (PU.1) bound regions $\left(z\right.$ score $=4.62, P=2 \times 10^{-4}$; Additional file 6: Figure S2).

For both macrophages and microglia, SPI1 (PU.1) motif-containing OCRs were significantly enriched, indicating that this class of OCRs is of relevance to $A D$ genetic risk mechanisms in both cell types. CEBP and MEF2 motif-containing OCRs survived correction for multiple testing in macrophages and were nominally significant (uncorrected $P<0.05$ ) in microglia. Several motifcontaining OCR sets were tested for only one cell type as de novo motif analysis did not identify them in the other, e.g. EGR1 for macrophages and RUNX for microglia.
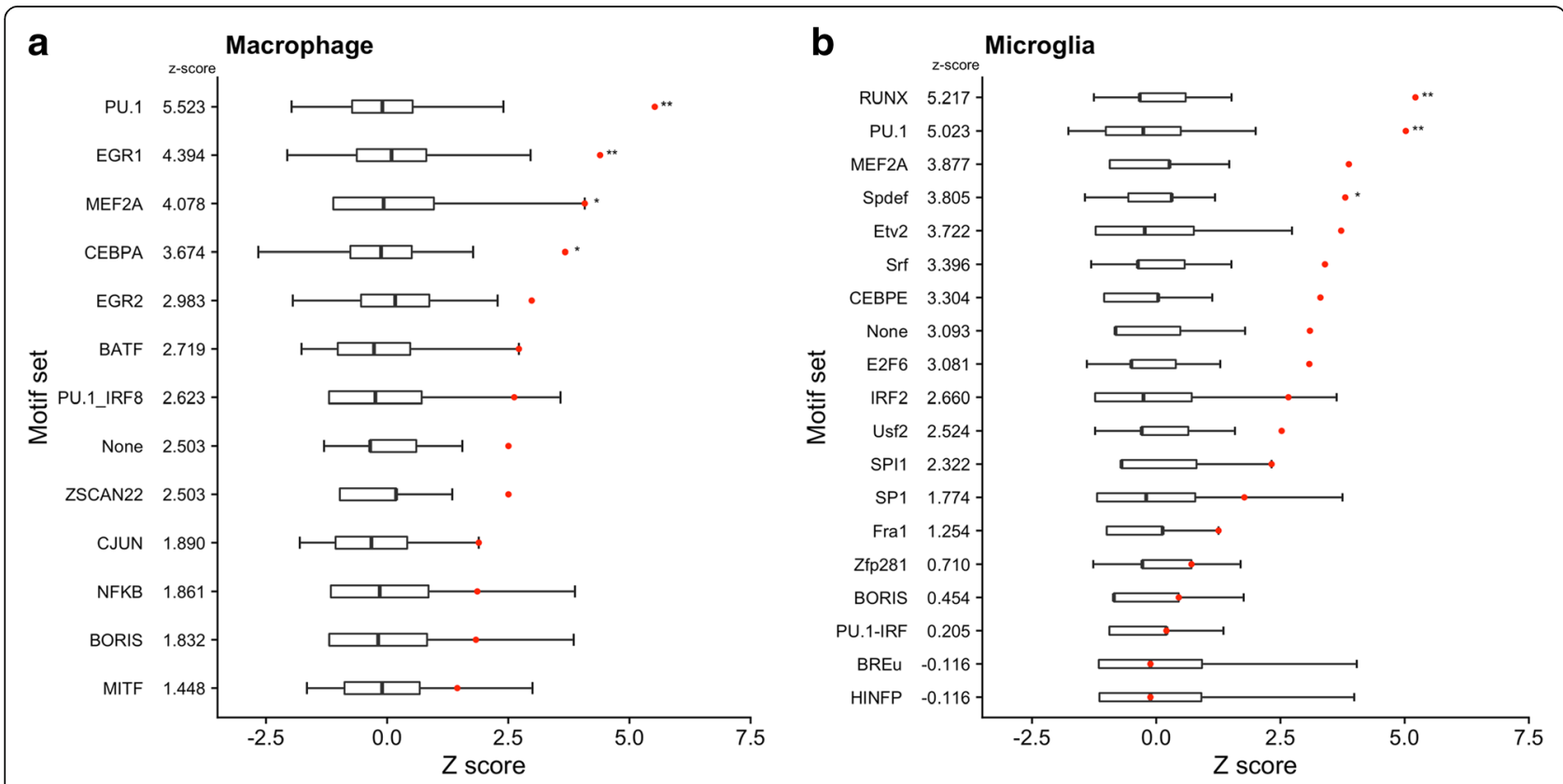

Fig. 4 Overlap between genome-wide significant AD risk variants and transcription factor motif-containing open chromatin sites from the consolidated macrophage (a) and microglia (b) data. AD risk variants and 10,000 sets of matched SNPs were intersected with transcription factor motif-containing open chromatin region sets and one no-motif-containing set for each cell type. The $x$-axis is the $z$ score and the $y$-axis is the transcription factor motif. Box plots indicate the distribution of overlap from the 10,000 background matched SNP sets. Red circles are the $z$ scores for the AD risk variant set. $P$ values were calculated from the observed overlap of the 10,000 background matched sets. $P$ values have been adjusted within each cell type using the Bonferroni correction for the number of motif sets tested. AD Alzheimer's disease, SNP single-nucleotide polymorphism. ${ }^{* *} P<0.005$, ${ }^{*} P<0.05$ 


\section{Common variant heritability of $A D$ is enriched at specific transcription factor motif-containing open chromatin regions}

Although many genome-wide significant AD risk loci have been identified, they account for a small proportion of the genetic heritability. Instead, thousands of variants across the entire genome collectively contribute to the polygenic inheritance of $\mathrm{AD}$. We reasoned that transcription factor motif-containing OCRs identified as being enriched at genome-wide significant loci would also be important for mediating polygenic inheritance. Therefore, we partitioned AD heritability by macrophage or microglia motif sets using LDscore regression [12].

Consistent with the macrophage SNP enrichment analysis of genome-wide significant loci, AD heritability was significantly enriched at variants in the DHS motif sets SPI1 (PU.1) (enrichment $=8.93$, corrected enrichment $P=0.012$ ), MEF2A (enrichment $=19.22$, corrected enrichment $P=0.022$ ), CEBPA (enrichment $=9.72$, corrected enrichment $P=3.43 \times 10^{-3}$ ) and EGR1 (enrichment $=14.48$, corrected enrichment $\left.P=5.14 \times 10^{-4}\right)$. $P$ values for all the transcription factors tested withstood Bonferroni correction for multiple testing (Table 1). Importantly, the no-motif DHS set was not significantly enriched (corrected enrichment $P=0.625$ ) (Table 1). Additional file 10: Table S9 contains the full results.

In microglia, $\mathrm{AD}$ heritability was significantly enriched at variants in the OCR motif sets SPI1 (PU.1) (enrichment = 16.28, corrected enrichment $P=4.39 \times 10^{-3}$ ) and Spdef (enrichment $=19.92$, corrected enrichment $P=0.040$ ). The RUNX OCR motif set was not significantly enriched (enrichment $=14.09$, correct enrichment $P=0.412$ ), nor was the no-motif set (enrichment $=20.27$, corrected enrichment $P=0.168)$. $P$ values were corrected using Bonferroni correction for multiple testing accounting for the number of tests undertaken within each cell type (Table 2). Additional file 11: Table S10 contains the full results. The enrichment of $\mathrm{AD}$ heritability at variants in SPI1 motif-containing OCRs was validated using the

Table 1 Enrichment of AD heritability at variants within EGR1, CEBPA, MEF2A, SPI1 and no-motif-containing macrophage DNase hypersensitivity sites

\begin{tabular}{llll}
\hline DHS motif set & Enrichment & Enrichment $P$ value & Corrected $P$ value \\
\hline EGR1 & 14.481 & $1.03 \times 10^{-4}$ & $5.14 \times 10^{-4}$ \\
CEBPA & 9.716 & $6.86 \times 10^{-4}$ & $3.43 \times 10^{-3}$ \\
SPI1 & 8.933 & $2.43 \times 10^{-3}$ & 0.012 \\
MEF2A & 19.222 & $4.46 \times 10^{-3}$ & 0.022 \\
No-motif & 7.661 & 0.125 & 0.623 \\
\hline
\end{tabular}

AD heritability was partitioned by transcription factor motif-containing DNase hypersensitivity sites using LDscore regression [12]. AD heritability was significantly enriched (after correcting for multiple testing) at all four transcription factor motif-containing DNase hypersensitivity sites but not the no-motif set $A D$ Alzheimer's disease
Table 2 Enrichment of AD heritability at variants within SPI1, Spdef, RUNX and no-motif-containing microglia ATAC-seq peaks

\begin{tabular}{llll}
\hline Motif set & Enrichment & Enrichment $P$ value & Corrected $P$ value \\
\hline SPI1 & 16.28 & $1.10 \times 10^{-3}$ & $4.39 \times 10^{-3}$ \\
Spdef & 19.92 & $9.93 \times 10^{-3}$ & 0.040 \\
No-motif & 20.27 & 0.042 & 0.168 \\
RUNX & 14.09 & 0.103 & 0.412
\end{tabular}

AD heritability was partitioned by transcription factor motif-containing ATAC-seq peaks using LDscore regression [12]. AD heritability was significantly enriched (after correcting for multiple testing) at SPI1 and Spdef transcription factor motif-containing ATAC-seq peaks but not the RUNX or no motif sets $A D$ Alzheimer's disease

SPI1 ChIP-seq data. Variants at these SPI1 bound regions were also substantially enriched for AD heritability (enrichment $=20.56$, enrichment $P=6.9 \times 10^{-4}$ ).

\section{Discussion}

Although GWAS have identified thousands of variants that influence diseases and traits, the majority are located in non-coding regions of the genome [2]. Combined with small effect sizes, the biological interpretation of these results is challenging. We have integrated results from GWAS of AD with OCRs identified in different tissue types, first by using genome-wide significant loci and then extending our analyses to genome-wide measurements of partitioned heritability. Through this two-stage approach, we identify alleles of potential functional significance that are amenable to further mechanistic investigation, and show variants contributing to polygenic inheritance are likely to operate through shared mechanisms. Specifically, these analyses identified macrophage and microglia transcriptional networks in which both genome-wide significant alleles and polygenic risk for $\mathrm{AD}$ are enriched.

The localisation of AD risk variants to DHSs from multiple immune cell types assayed by the Epigenomics Roadmap Project highlights their potential importance in mediating the effects of $\mathrm{AD}$ genetic risk, and is in agreement with other studies $[4,5]$. Our analyses using histone modifications that are indicative of active gene regulatory elements, also strongly supports the role of immune cells, particularly monocytes, in $\mathrm{AD}$ genetic risk mechanisms. Enrichment at all three histone modifications tested suggests risk mechanisms involve multiple types of regulatory elements (e.g. promoters and enhancers). Combined with results generated using data from the Blueprint Epigenome Project, we provide replicated evidence for the enrichment of AD risk variants at monocyte DHSs. A similar enrichment is also observed at macrophage DHSs, a cell type derived from monocytes that have invaded a target tissue. Finally, we show that microglia are also plausibly linked to $\mathrm{AD}$ genetic risk mechanisms via regions of open chromatin. Unlike 
studies of post-mortem material, where cause cannot easily be separated from consequence, genetic associations do not suffer from problems of reverse causation. Therefore, our findings implicate immune cell dysfunction as a causal factor in AD risk. Given the extensive overlap between regulatory elements in related cell types, it is not currently possible to identify a single causal immune cell type and we cannot exclude the involvement of multiple cell types in AD risk mechanisms. However, the location of microglia in the brain positions them as the likely causal candidates.

In contrast to the significant enrichment at immune cell DHSs, AD risk variants were not enriched at brain DHSs identified using bulk tissue. However, DHSs data from the Epigenomics Roadmap Project contains only two brain samples, both foetal. The enrichment at microglial OCRs suggests that they are the plausible brain cell type in which AD risk mechanisms operate, and that profiles from bulk tissue suffer from lack of cell-type specificity. It is necessary to investigate additional brain data as they become available, particularly those that can resolve cell-type specific information [26]. It should also be noted that the currently available data are primarily derived from healthy donors under basal conditions. To investigate the gene regulatory mechanisms underlying genetic disease risk fully, it may be necessary to investigate cells under a variety of conditions, including those thought to be environmental risk factors for disease.

Consistent with gene-based pathway analysis of $\mathrm{AD}$ GWAS [27, 28], these loci harbour genes such as $P T K 2 B$ and INPP5D that encode for proteins with recognised immune functions and have immune cell-type enriched expression. However, it is at the level of DNA regulatory elements that tissue-specific risk mechanisms are generated. Indeed, our analysis identifies a number of ubiquitously expressed genes (e.g. BIN1 and $C D 2 A P$ ) at which $\mathrm{AD}$ associated risk variation could credibly operate in immune cells, including microglia. Therefore, the number of AD risk loci that impact on immune cell function is likely to be larger than that captured by current genebased pathway annotation methods. It is now important to identify the biological processes that are disrupted by $\mathrm{AD}$ risk variants in immune cells.

Of the 18 genome-wide significant loci tested, 13 have at least one variant located in a macrophage DHS and 11 in a microglial OCR, indicating that the majority of $\mathrm{AD}$ risk loci plausibly operate to alter gene expression in these cells. At most of these loci, more than one SNP overlapped an OCR, suggesting that individual risk loci are likely to harbour multiple functional variants. By focusing on OCRs containing transcription factor motifs, the number of overlapping SNPs at each locus is reduced. For example, in microglia, eight of the 11 loci contain three or fewer SNPs overlapping a SPI1 motifcontaining OCR. These variants can, therefore, be prioritised for further molecular characterisation.

Having established an enrichment of AD risk variants at macrophage and microglia OCRs, we investigated their localisation to OCRs containing motifs for specific transcription factors. Within a given cell type, thousands of transcriptional regulators contribute to the control of gene expression, but master regulators, often cell type specific, can be recovered by a motif analysis of regulatory element sequences. In both macrophage and microglia, AD risk variants were enriched at OCRs containing specific transcription factor motifs, supporting the hypothesis that risk variants are localised to specific transcription factor targeted OCRs, including experimentally determined SPI1 bound regions in microglia.

Of particular interest is the enrichment of $\mathrm{AD}$ risk SNPs at SPI1 and MEF2A motif-containing OCRs. Genetic variants at, or in close proximity to, SPI1 and MEF2C (HOMER reports that the MEF2C and MEF2A motifs have a similarity score of 0.94 ) have been identified as significant AD risk loci [1, 29]. Impaired transcriptional control by these factors, either through altered gene expression in cis or via disrupted DNA binding due to genetic variants at target sites, is likely to play a central role in AD genetic risk mechanisms. The importance of variants in these motif-containing OCRs extends beyond those reaching genome-wide significance, providing evidence that the thousands of subthreshold variants contributing to polygenic risk collectively operate by similar mechanisms. Although enrichment at these sites is large ( 9-19 fold), and account for a substantial proportion of the total SNP-chip heritability, the $P$ values reported are weaker than those observed in analyses of some other diseases using chromatin features [12]. This is most likely due to the low SNP-chip heritability of AD as calculated by LDscore regression ( 7\%, http://ldsc.broadinstitute.org/lookup/). GWAS data from larger cohorts will be important for defining risk mechanisms at increased molecular resolution. Similarly, the identification of transcription factor motifs from studies of open chromatin derived from additional methods will reduce potential single source biases.

More generally, our results support a model of polygenic disease risk that is enriched at defined transcriptional networks operating in cell types relevant to disease. For other complex disorders such as type 2 diabetes, genome-wide significant risk variants have been shown to localise to specific transcription factor binding sites in islet cells $[30,31]$, but the extent to which variants in these binding sites contribute to polygenic inheritance was not investigated. We show that polygenic risk arising from non-coding variation is localised to specific transcription factor networks. For AD, this is most 
prominent for a potential SPI1-driven network, consistent with a targeted investigation [32].

SPI1 encodes a transcription factor known to be critical for the development and function of haematopoietic cell lineages [33], including microglia [25]. Decreased expression of SPI1 and CEBPA (also identified through motif enrichment analysis in macrophages) is observed after a reduction in AD-like pathology and behaviour in APPswe/ PSEN1dE9 mice following pharmacological inhibition of the receptor CSF1R [34]. Therefore, our results link polygenic $\mathrm{AD}$ risk mechanisms to transcriptional networks that have therapeutic validity. The identification of upstream regulators of these transcription factors may yield novel targets that are important for AD therapies.

\section{Conclusions}

In summary, integration of GWAS results with sites of open chromatin identifies immune cells as likely mediators of common variant genetic risk for $\mathrm{AD}$. The majority of genome-wide significant $\mathrm{AD}$ risk loci plausibly operate in peripheral monocytes, macrophages and/or microglia, and we identify candidate SNPs at these loci suitable for targeted mechanistic studies based on shared OCR annotations. Within open chromatin sites, those containing specific DNA motifs drive this enrichment. Similarly, genetic variants at these sites capture a substantial proportion of the AD common variant SNP-chip heritability, $67 \%$ for the SPI1 targeted sites, increasing the molecular resolution of $\mathrm{AD}$ genetic risk mechanisms from cell type to transcriptional networks. We provide evidence for the causal role of microglia in $\mathrm{AD}$ pathogenesis and therefore, a parsimonious explanation for the involvement of immune cells in $\mathrm{AD}$ risk mechanisms. Furthermore, we establish that the thousands of variants contributing to $\mathrm{AD}$ polygenic risk are enriched at specific macrophage/microglial transcriptional networks, placing them in tangible biological pathways amenable to future mechanistic studies.

\section{Additional files}

Additional file 1: Table S1. Enrichment of AD-risk variants at DHSs from Roadmap Epigenomics Consortium data. (XLSX 36 kb)

Additional file 2: Table S2. Enrichment of AD-risk variants at H3K27ac peaks from Roadmap Epigenomics Consortium data. (XLSX 54 kb)

Additional file 3: Table S3. Enrichment of AD-risk variants at H3K4me1 peaks from Roadmap Epigenomics Consortium data. (XLSX 56 kb)

Additional file 4: Table S4. Enrichment of AD-risk variants at H4K3me3 peaks from Roadmap Epigenomics Consortium data. (XLSX $48 \mathrm{~kb}$ )

Additional file 5: Table S5. AD-risk SNPs overlapping macrophage DHS. (XLSX $10 \mathrm{~kb}$ )

Additional file 6: Figure S1. Open chromatin regions (OCRs) identified in macrophages and microglia at Alzheimer's disease risk loci containing the genes BIN1 and CASS4. Figure S2. Overlap between Alzheimer's disease risk loci and genomic regions bound by SPI1 in human ex vivo microglia. (PDF $595 \mathrm{~kb}$ )
Additional file 7: Table S6. AD-risk SNPs that overlap microglia ATAC-seq peaks. (XLSX $12 \mathrm{~kb}$ )

Additional file 8: Table S7. Results from the de novo motif analysis performed using HOMER on the macrophage DHS. (XLSX $10 \mathrm{~kb}$ )

Additional file 9: Table S8. Results from the de novo motif analysis performed using HOMER on microglia ATAC-seq peaks. (XLSX $10 \mathrm{~kb}$ )

Additional file 10: Table S9. Enrichment of $A D$ heritability at variants within the motif-containing macrophage DHS. (XLSX 9 kb)

Additional file 11: Table S10. Enrichment of AD heritability at variants within motifs containing microglia ATAC-seq peaks. (XLSX $30 \mathrm{~kb}$ )

\section{Abbreviations}

AD: Alzheimer's disease; ATAC-seq: Assay for transposase-accessible chromatin using sequencing; ChIP: Chromatin immunoprecipitation; DHS: DNase hypersensitivity site; GWAS: Genome-wide association studies; LD: Linkage disequilibrium; MHC: Major histocompatibility complex; OCR: Open chromatin region; SNP: Single nucleotide polymorphism

\section{Acknowledgements}

This study makes use of data generated by the Blueprint Consortium. A full list of the investigators who contributed to the generation of the data is available from www.blueprint-epigenome.eu. Funding for the project was provided by the European Union's Seventh Framework Programme (FP7/2007-2013) under grant agreement 282510 (Blueprint).

\section{Funding}

KET is supported by the Wellcome Trust (grant reference: WT105613/Z/14/Z) and the College of Biomedical and Life Sciences at Cardiff University. DC is supported by an Medical Research Council PhD studentship (MR/L010305/1).

\section{Availability of data and materials}

The data sets analysed during the current study are available from Roadmap Epigenomics Project (http://egg2.wustl.edu/roadmap/data/byFileType/peaks/ consolidated/broadPeak/DNase/; http://egg2.wustl.edu/roadmap/data/ byFileType/peaks/consolidated/broadPeak/; http://egg2.wustl.edu/roadmap/ data/byFileType/peaks/consolidated/narrowPeak/), BLUEPRINT (http://ftp. ebi.ac.uk/pub/databases/blueprint/data/homo_sapiens/GRCh37/), dbGAP (https://www.ncbi.nlm.nih.gov/projects/gap/cgi-bin/study.cgi?study_ id=phs001373.v1.p1), IGAP (http://web.pasteur-lille.fr/en/recherche/u744/igap/ igap_download.php) and 1000 Genomes (http://www.internationalgenome. org/about\#ProjectSamples).

\section{Authors' contributions}

$\mathrm{MJH}$ conceived the project. KET and $\mathrm{MJH}$ analysed the data and wrote the manuscript. DC processed and analysed the microglia data. All authors read and approved the final manuscript.

Ethics approval and consent to participate Not applicable.

\section{Consent for publication}

Not applicable.

Competing interests

The authors declare that they have no competing interests.

\section{Publisher's Note}

Springer Nature remains neutral with regard to jurisdictional claims in published maps and institutional affiliations.

\section{Author details}

${ }^{1}$ Core Bioinformatics and Statistics Team, College of Biomedical and Life Sciences, Cardiff University, Cardiff, UK. ${ }^{2}$ MRC Centre for Neuropsychiatric Genetics and Genomics, Division of Psychological Medicine and Clinical Neurosciences, School of Medicine, College of Biomedical and Life Sciences, Cardiff University, Hadyn Ellis Building, Maindy Road, Cardiff CF24 4HQ, UK. ${ }^{3}$ UK Dementia Research Institute, Cardiff University, Hadyn Ellis Building, Maindy Road, Cardiff CF24 4HQ, UK. 
Received: 2 November 2017 Accepted: 14 February 2018 Published online: 26 February 2018

\section{References}

1. Lambert JC, Ibrahim-Verbaas CA, Harold D, Naj AC, Sims R, Bellenguez C, et al. Meta-analysis of 74,046 individuals identifies 11 new susceptibility loci for Alzheimer's disease. Nat Genet. 2013;45:1452-8. https:/doi.org/10.1038/ng.2802.

2. Schaub MA, Boyle AP, Kundaje A, Batzoglou S, Snyder M. Linking disease associations with regulatory information in the human genome. Genome Res. 2012;22:1748-59. https://doi.org/10.1101/gr.136127.111.

3. Jones L, Lambert J-C, Wang L-S, Choi S-H, Harold D, Vedernikov A, et al. Convergent genetic and expression data implicate immunity in Alzheimer's disease. Alzheimer's Dement. 2015;11:658-71. https://doi.org/10.1016/j.jalz. 2014.05.1757.

4. Gjoneska E, Pfenning AR, Mathys H, Quon G, Kundaje A, Tsai L-H, et al. Conserved epigenomic signals in mice and humans reveal immune basis of Alzheimer's disease. Nature. 2015;518:365-9. https://doi.org/10.1038/ nature14252.

5. Gagliano SA, Pouget JG, Hardy J, Knight J, Barnes MR, Ryten M, et al. Genomics implicates adaptive and innate immunity in Alzheimer's and Parkinson's diseases. Ann Clin Transl Neurol. 2016;3:924-33. https://doi.org/ 10.1002/acn3.369.

6. Dunham I, Kundaje A, Aldred SF, Collins PJ, Davis CA, Doyle F, et al. An integrated encyclopedia of DNA elements in the human genome. Nature. 2012;489:57-74. https://doi.org/10.1038/nature11247.

7. Kundaje A, Meuleman W, Ernst J, Bilenky M, Yen A, Heravi-Moussavi A, et al. Integrative analysis of 111 reference human epigenomes. Nature. 2015:518:317-30. https://doi.org/10.1038/nature14248.

8. Maurano MT, Humbert R, Rynes E, Thurman RE, Haugen E, Wang H, et al. Systematic localization of common disease-associated variation in regulatory DNA. Science. 2012;337:1190-5. https://doi.org/10.1126/science.1222794.

9. Won $\mathrm{H}-\mathrm{H}$, Natarajan $\mathrm{P}$, Dobbyn A, Jordan DM, Roussos P, Lage K, et al. Disproportionate contributions of select genomic compartments and cell types to genetic risk for coronary artery disease. PLoS Genet. 2015;11:e1005622. https://doi.org/10.1371/journal.pgen.1005622.

10. Yang J, Benyamin B, McEvoy BP, Gordon S, Henders AK, Nyholt DR, et al. Common SNPs explain a large proportion of the heritability for human height. Nat Genet. 2010;42:565-9. https://doi.org/10.1038/ng.608.

11. Bulik-Sullivan BK, Loh P-R, Finucane HK, Ripke S, Yang J, Patterson N, et al. LD Score regression distinguishes confounding from polygenicity in genome-wide association studies. Nat Genet. 2015;47:291-5. https://doi.org/10.1038/ng.3211.

12. Finucane HK, Bulik-Sullivan B, Gusev A, Trynka G, Reshef $Y$, Loh P-R, et al. Partitioning heritability by functional annotation using genome-wide association summary statistics. Nat Genet. 2015;47:1228-35. https://doi.org/10.1038/ng.3404.

13. Boyle AP, Davis S, Shulha HP, Meltzer P, Margulies EH, Weng Z, et al. High-resolution mapping and characterization of open chromatin across the genome. Cell. 2008;132:311-22. https://doi.org/10.1016/j.cell.2007.12.014.

14. Buenrostro JD, Giresi PG, Zaba LC, Chang HY, Greenleaf WJ. Transposition of native chromatin for fast and sensitive epigenomic profiling of open chromatin, DNA-binding proteins and nucleosome position. Nat Methods. 2013;10:1213-8. https://doi.org/10.1038/nmeth.2688.

15. Song L, Zhang Z, Grasfeder LL, Boyle AP, Giresi PG, Lee B-K, et al. Open chromatin defined by DNasel and FAIRE identifies regulatory elements that shape cell-type identity. Genome Res. 2011;21:1757-67. https://doi.org/10. 1101/gr.121541.111.

16. Kaser A, Zeissig S, Blumberg RS, Asl HF, Hijma HJ, Cuppen EPJG, et al. Inflammatory bowel disease. Annu Rev Immunol. 2010;28:573-621. https://doi.org/10.1146/annurev-immunol-030409-101225.

17. Quinlan AR, Hall IM. BEDTools: a flexible suite of utilities for comparing genomic features. Bioinformatics. 2010;26:841-2. https://doi.org/10.1093/ bioinformatics/btq033.

18. Gosselin D, Skola D, Coufal NG, Holtman IR, Schlachetzki JCM, Sajti E, et al. An environment-dependent transcriptional network specifies human microglia identity. Science. 2017;356:1248-59. https://doi.org/10.1126/ science.aal3222.

19. Li H, Durbin R. Fast and accurate short read alignment with Burrows-Wheeler transform. Bioinformatics. 2009;25:1754-60. https://doi.org/10.1093/ bioinformatics/btp324.
20. John S, Sabo PJ, Thurman RE, Sung M-H, Biddie SC, Johnson TA, et al. Chromatin accessibility pre-determines glucocorticoid receptor binding patterns. Nat Genet. 2011;43:264-8. https://doi.org/10.1038/ng.759.

21. MacArthur J, Bowler E, Cerezo M, Gil L, Hall P, Hastings E, et al. The new NHGRIEBI Catalog of published genome-wide association studies (GWAS Catalog). Nucleic Acids Res. 2017:45:D896-901. https://doi.org/10.1093/nar/gkw1133.

22. Pers TH, Timshel P, Hirschhorn JN. SNPsnap: a web-based tool for identification and annotation of matched SNPs. Bioinformatics. 2015;31:418-20. https://doi.org/10.1093/bioinformatics/btu655.

23. Heinz S, Benner C, Spann N, Bertolino E, Lin YC, Laslo P, et al. Simple combinations of lineage-determining transcription factors prime cis-regulatory elements required for macrophage and B cell identities. Mol Cell. 2010;38:576-89. https://doi.org/10.1016/j.molcel.2010.05.004

24. Benjamini $Y$, Hochberg $Y$. Controlling the false discovery rate: a practical and powerful approach to multiple testing. J R Stat Soc Ser B (Methodol). 1995;57:289-300. https://doi.org/10.2307/2346101.

25. Kierdorf K, Erny D, Goldmann T, Sander V, Schulz C, Perdiguero EG, et al. Microglia emerge from erythromyeloid precursors via Pu.1- and Irf8-dependent pathways. Nat Neurosci. 2013;16:273-80. https://doi.org/10.1038/nn.3318.

26. Jiang Y, Matevossian A, Huang H-S, Straubhaar J, Akbarian S, Tsankova N, et al. Isolation of neuronal chromatin from brain tissue. BMC Neurosci. 2008:9:42. https://doi.org/10.1186/1471-2202-9-42.

27. Zhang B, Gaiteri C, Bodea L-G, Wang Z, McElwee J, Podtelezhnikov AA, et al. Integrated systems approach identifies genetic nodes and networks in late-onset Alzheimer's disease. Cell. 2013;153:707-20. https://doi.org/ 10.1016/j.cell.2013.03.030

28. Jones L, Holmans PA, Hamshere ML, Harold D, Moskvina V, Ivanov D, et al Genetic Evidence Implicates the Immune System and Cholesterol Metabolism in the Aetiology of Alzheimer's Disease. PLoS One. 2010;5: e13950. https://doi.org/10.1371/journal.pone.0013950.

29. Escott-Price V, Bellenguez C, Wang L-S, Choi S-H, Harold D, Jones $L$, et al. Gene-wide analysis detects two new susceptibility genes for Alzheimer's disease. PLoS One. 2014;9:e94661. https://doi.org/10.1371/journal.pone. 0094661.

30. Varshney A, Scott LJ, Welch RP, Erdos MR, Chines PS, Narisu N, et al. Genetic regulatory signatures underlying islet gene expression and type 2 diabetes. Proc Natl Acad Sci. 2017:201621192. https://doi.org/10.1073/pnas.1621192114.

31. Gaulton KJ, Ferreira T, Lee Y, Raimondo A, Mägi R, Reschen ME, et al. Genetic fine mapping and genomic annotation defines causal mechanisms at type 2 diabetes susceptibility loci. Nat Genet. 2015;47:1415-25. https://doi.org/10.1038/ng.3437.

32. Huang K, Marcora E, Pimenova AA, Di Narzo AF, Kapoor M, Jin SC, et al. A common haplotype lowers PU.1 expression in myeloid cells and delays onset of Alzheimer's disease. Nat Neurosci. 2017;20:1052-61. https://doi.org/ 10.1038/nn.4587.

33. McKercher SR, Torbett BE, Anderson KL, Henkel GW, Vestal DJ, Baribault H, et al. Targeted disruption of the PU.1 gene results in multiple hematopoietic abnormalities. EMBO J. 1996;15:5647-58. https://doi.org/10.1002/j.1460-2075. 1996.tb00949.x.

34. Olmos-Alonso A, Schetters STT, Sri S, Askew K, Mancuso R, Vargas-Caballero M, et al. Pharmacological targeting of CSF1R inhibits microglial proliferation and prevents the progression of Alzheimer's-like pathology. Brain. 2016;139(Pt 3):891-907. https://doi.org/10.1093/brain/awv379.

\section{Submit your next manuscript to BioMed Central and we will help you at every step:}

- We accept pre-submission inquiries

- Our selector tool helps you to find the most relevant journal

- We provide round the clock customer support

- Convenient online submission

- Thorough peer review

- Inclusion in PubMed and all major indexing services

- Maximum visibility for your research

Submit your manuscript at www.biomedcentral.com/submit 